\title{
Isolated palatal injury due to a bicycle accident
}

SADJ October 2018, Vol. 73 No. 9 p560 - p562

M Mabongo', K Sukha²

\section{ABSTRACT}

Trauma to the soft palate due to impalement of a bicycle handlebar does not appear to have been reported in the literature. More commonly the trauma occurs in toddlers due to impalement with objects such as pencils. We report an atypical presentation of an 11 year old with a significantly lacerated soft palate suffered when he fell off a bicycle.

The child's mouth must have been open when he fell, which allowed the handlebar to injure the soft palate without causing damage to his teeth. A clinical evaluation and advanced radiographic images revealed significant impairment of the musculature of the soft palate.

Treatment was performed under general anaesthesia and involved correct placement and suturing of these anatomical structures, which were ectopically displaced.

Good oral hygiene was prescribed, including the use of a chlorhexidine based oral rinse. The patient's recovery was uneventful with almost complete healing within three weeks.

It is clear that bicycle safety needs more emphasis in South Africa. Apart from the use of safety devices such as helmets and mouth guards, there may be a need for legislation to be amended to enhance safe accommodation on the roads for cyclists.

\section{INTRODUCTION}

Injuries associated with the childhood achievement of bicycle riding can have devastating effects on both victims and parents. An estimated 900 deaths and 494,000 visits to emergency department occurred due to bicycle-related injuries in the USA, translating into an expenditure of over $\$ 2$ billion on bicycle-related traumatic brain injuries (TBI). ${ }^{1}$

Brain and maxillofacial trauma, and dental injuries are common amongst bicycle riders. ${ }^{1}$ Bicycle accidents may also result in trauma to the abdominal organs including the liver, spleen and kidney.

Author affiliations:

1. Mzubanzi Mabongo, STD (Unitra), B Dent (Medunsa), BDS Wits), MChD (MFOS)(UWC), Cert. Orofacial pain (Rutgers, USA), FCFS (Bangalore, India), Head of Clinical Unit at Charlotte Maxeke Academic Hospital, Department of Maxillo-Facial and Oral Surgery,

University of the Witwatersrand, Johannesburg.

2. Kamal Sukha, BDS (Wits), MSc (Dent) (Wits),

Dentist in Private Practice

Corresponding author: Kamal Sukha

P.O. Box 709, Lenasia, 1820.

Tel: +27 (0)112114110 Email: dr.ksukha@gmail.com

\section{ACRONYMS}

$\begin{array}{ll}\text { BSI: } & \text { Bicycle Spoke Injury } \\ \text { MIO: } & \text { Maximal Interincisal Opening } \\ \text { MRI: } & \text { Magnetic Resonance Image } \\ \text { TBI: } & \text { Traumatic Brain Injuries }\end{array}$

The thorax, genito-urinary organs and limbs are also at risk. ${ }^{2}$ Bicycle spoke injury (BSI) involves the foot, ankle or the lower leg as a result of entrapment in the bicycle spokes, while bicycle handlebar injury may involve abdominal trauma. TBI is the leading cause of morbidity and mortality after bicycle-related accidents.

Maxillofacial trauma resulting from bicycle injuries includes orbital, zygomatic, nasal and mandibular fractures. ${ }^{2,3}$ Dental injuries include crown or root fracture, luxation, intrusion and avulsion. ${ }^{3}$

Most injuries involve superficial trauma such as abrasions ("road rash"), contusions and lacerations. Abrasions can range from being superficial to those involving partial or full skin thickness. Treatment requires debridement and irrigation of embedded residue to prevent "traumatic tattooing". ${ }^{4}$

Cyclists may suffer accidental falls as a result of inattention, poor road conditions, and road traffic accidents such as collisions with other cyclists or motor vehicles. ${ }^{3}$ To our knowledge, isolated injuries of the soft palate due to bicycle accidents have not been reported in the literature.

\section{CASE REPORT}

An 11 year-old boy was referred to the practice by a medical doctor a week after he had fallen from a bicycle. The initial consultation had been at a local district clinic where analgesics and a mouth rinse were prescribed.

A clinical assessment revealed trismus with a maximal interincisal opening (MIO) of $30 \mathrm{~mm}$. He complained of dysphagia and dysphonia while hypernasal speech was also noted.

The temporomandibular joints were not tender. Both the mandible and maxilla were intact and there was no neurological deficit of the face. An intraoral examination showed a laceration of approximately $2 \mathrm{~cm}$ on the right side of the hard palate, compounded by a significant soft palatal defect.

There was also a previous wound on the posterior end of the right hard palate which extended to the midline, and posteriorly to the uvula. The entire right side of 
the soft palate was detached anteriorly and medially (Figure 1a and 1b). The right tonsil appeared to be enlarged. A magnetic resonance image (MRI) was requested to determine the orientation of the residual soft palatal muscle.

The MRI revealed a soft palatal defect which measured $16,6 \mathrm{~mm}$ in a craniocaudal dimension and $9 \mathrm{~mm}$ in an anterior-posterior dimension. Asymmetric enlargement of the palatine tonsil was also observed. Diffuse high signal disturbances were noted in the right veli palatini muscles. Defects were also noted in both the palatopharyngeus and palatoglossus muscles. Intense inflammation was present around the defect.

The patient was taken to theatre for an examination under anaesthesia and reconstruction of the remaining soft tissues of the soft palate.

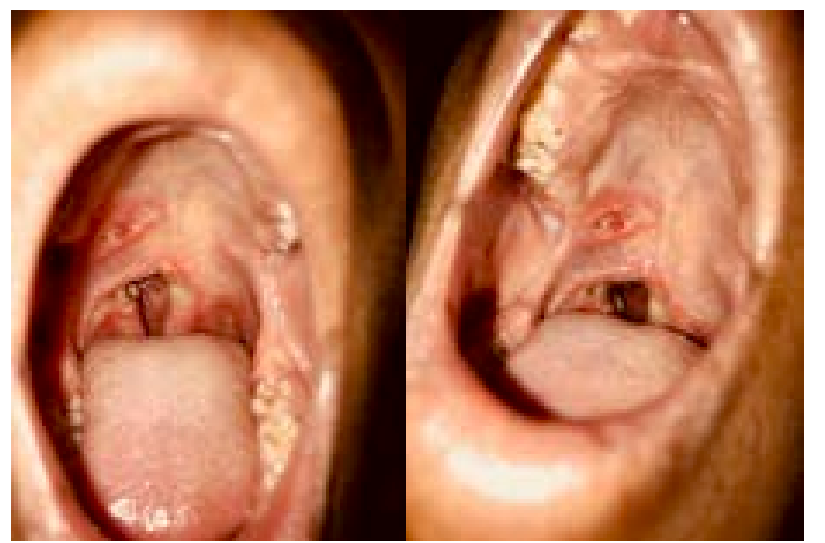

Figure 1a. Figure 1b.

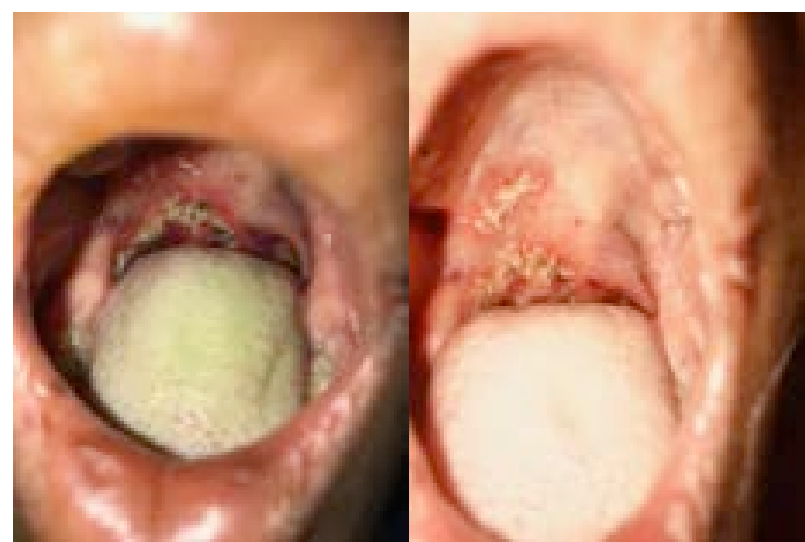

Figure 2a. Figure 2b.

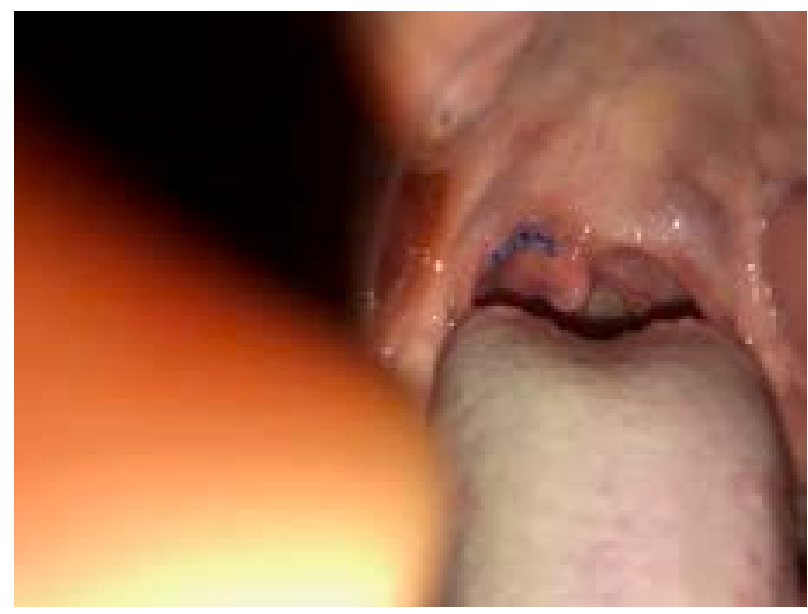

Figure 3.
Possible modification of the treatment plan included harvesting a right buccal mucomyocutaneous flap if there was inadequate viable tissue for reconstruction.

On examination, what had seemed like an enlarged right tonsil was found to actually be the soft palate, suspended into the oropharynx and healing in an ectopic position as it was still attached to the right lateral oropharynx. The treatment plan was modified to include mobilisation of the detached soft palate.

All wound margins were debrided and sutured in three layers, the nasal mucosa, muscle and oral mucosa. The patient recovered without any complications from the procedure and the general anaesthesia. He was discharged with strict instructions that included a soft diet and the use of an oral rinse with a 0,2\% chlorhexidine gluconate solution.

A postoperative examination of the repaired tissues performed a day (Figure 2a) and one week after the procedure (Figure $2 \mathrm{~b}$ ) revealed intact operative sites with no significant complications.

Clinical examination at the final review was performed three weeks postoperatively, at which time the soft palate had almost completely healed (Figure 3). The patient also reported that his vocal ability was restored (no signs of hypernasal speech) and the pain and dysphagia had settled.

\section{DISCUSSION}

Maxillo-facial injuries related to bicycle falls are often quite severe. Junior et al described mandibular fractures as being the most common facial bone fracture followed by zygomatic complex fractures in both motorcycle and bicycle falls in Brazil. ${ }^{5}$

An Italian paper reported that facial and dental injuries related to bicycle accidents included crown fractures, periodontal injuries, maxillo-facial bone fractures as well as soft tissue lacerations and contusions. ${ }^{6} \mathrm{~A}$ relevant finding noted by these authors was that facial fractures more frequently involved the mid-face in younger individuals, whilst the mandible, maxilla and zygoma suffered in slightly older children.

This significant finding could be credited to a larger, more developed and therefore a more susceptible mandible in these children. Facial fractures were often compounded by dental injuries.

The goals of the managing this patient were to restore form and function. The hypernasal speech could be explained by a loss of oropharyngeal seal resulting in escape of the air via the right soft palate defect to the nose during speech while the dysphagia was due to the exposed sensory nerve fibres of the open wound as well as the intense inflammation around the defect.

We hypothesise that the patient fell forward over the bicycle with his mouth widely opened during the accident, resulting in tearing of the soft palate by impact with the handlebar. 
Although the muscles of the soft palate were not dissected out during the repair, function and form were restored to the satisfaction of the parents and the patient. Assessment of the soft palate revealed a fully functional levator veli palatini muscle. Although rare, an injury of this nature should not be treated casually.

Caldwell described an incident of a young male who suffered an impalement injury to the soft palate. He then presented with neurological deficits and died within six days.

The cause was due to the formation of a post-traumatic intramural thrombus in the internal carotid artery (which lies in close proximity to the posterolateral oropharynx), which then resulted in a haemorrhagic stroke possibly due to embolization of the thrombus to the middle cerebral artery in the Circle of Willis. ${ }^{6}$

\section{CONCLUSION}

Bicycle riding is accompanied by a high risk of injury. Safety precautions such as helmet usage are of pivotal importance in terms of prevention. Helmets provide protection against severe TBI, reduces the possibility of facial fractures and saves lives even after the victim sustains an intracranial haemorrhage. ${ }^{7}$

Skull fractures and subdural haematomas are less prevalent in helmet wearers. ${ }^{8}$ Noakes found that in South Africa an estimated $85 \%$ of cycling fatalities due to head injury could have been prevented by helmet usage. ${ }^{8}$

He also stated that the frequency of wearing helmets amongst younger individuals was exceedingly low and therefore recommended that the drafting of appropriate legislature making helmet-wearing compulsory may be the only method of increasing usage.

Maxillo-facial injuries would still be prevalent even with helmet usage and it would therefore be sensible for bicycle users to embrace the wearing of mouth guards to provide some protection for dental and oral tissues. Bicycles equipped with retractable handlebars are also advantageous.

The wearing of reflective gear is strongly recommended, particularly for night cycling. ${ }^{9}$ Prevention should also ideally centre on education, supervision and the improvement of infrastructure. Learning programs at schools and community centres should focus on knowledge of road safety and cycling skills from a very young age.

The upgrading of rural roads and the designation of specific cycling lanes may seem to be an unrealistic short-term goal in South Africa but it is definitely an achievement to strive toward.

\section{References:}

1. Joseph B, Azim A, Haider A, et al. Bicycle helmets work when it matters the most. Am J Surg. 2017; 213: 413-17.

2. Klin B, Rosenfeld-Yehoshua N, Abu-Kishk I, et al. Bicycle related injuries in children: disturbing profile of a growing problem. Injury 2009; 40: 1011-13.

3. Boffano P, Roccia F, Gallesio C, et al. Bicycle-related maxillofacial injuries: a double-center study. Oral Surg Oral Med Oral Pathol Oral Radiol. 2013; 116: 275-80.

4. Rivara FP, Thompson DC, Patterson MQ, et al. Prevention of bicycle-related injuries: helmets, education and legislation. Annu Rev Public Health. 1998; 19: 293-318.

5. Lima Junior S, Santos S, Kluppel L, et al. A comparison of motorcycle and bicycle accidents in oral and maxillofacial trauma. Journal of Oral and Maxillofacial Surgery 2012; 70: 577-83.

6. Amadori F, Bardellini E, Copeta A, et al. Dental trauma and bicycle safety: a report in Italian children and adolescents. Acta Odontologica Scandinavica 2017; 75: 227-31.

7. David A, Randall D, Kang R. Current management of penetrating injuries of the soft palate. Otolaryngology-Head and Neck Surgery 2006; 135: 356-60.

8. Forbes AE, Schutzer-Weissmann J, Menassa DA, et al. Head injury patterns in helmeted and non-helmeted cyclists admitted to a London Major Trauma Centre with serious head injury. Plos 1 2017; 12: e0185367.

9. Noakes TD. Fatal cycling injuries. Sports Med. 1995; 20: 348-62.

10. Koekemoer K, Van Gesselleen M, Van Niekerk A, et al. Child pedestrian safety knowledge, behaviour and road injury in Cape Town, South Africa. Accid Anal Prev. 2017; 99: 202-9. 